\title{
08 MANAGEMENT OF VICTIMISED PATIENTS IN PRIMARY CARE SETTINGS: A COMPREHENSIVE GREEK EDUCATIONAL INITIATIVE
}

doi:10.1136/injuryprev-2012-040590k.8

${ }^{1} \mathrm{E}$ Petridou, ${ }^{2} \mathrm{M}$ Papadakaki, ${ }^{2} \mathrm{D}$ Prokopiadou, ${ }^{3} \mathrm{M}$ Kogevinas, ${ }^{2} \mathrm{C}$ Lionis. ${ }^{1}$ Department of Hygiene, Epidemiology and Medical Statistics, Athens University, Medical School, Greece; ${ }^{2}$ Clinic of Social and Family Medicine, Faculty of Medicine, University of Crete, Greece; ${ }^{3}$ National School of Public Health, Greece

Background Primary care providers have been criticised for inadequate routine assessment of patients' exposure to intimate partner violence (IPV).

Aims To report on the content/ methods used and experiences learned regarding three key objectives in identifying and managing victimised patients at primary care settings, as part of a Thesis.

Methods A qualitative study employing three focus groups with 18 General Practitioners drawn from respective Networks in Greece was designed to explore physicians' views, perceptions and current practices. Tools for the assessment of physicians' training needs were validated and implemented focusing on Physicians' Readiness to Manage Intimate Partner Violence (PREMIS) employing a sample of 80 physicians. Lastly, acceptability and effectiveness of an educational outreach aiming at increasing physicians' perceived and actual knowledge, perceived readiness and number of detected cases was assessed using a pre/post-evaluation study design of a two-day training programme offered to 26 licensed General Practitioners/General Practice residents employing also a control group.

Results Issues of discomfort in discussing IPV, lack of confidence in diagnoses and mistrust in referral structures were indicated as barriers of effective case management. The Greek version of the PREMIS survey demonstrated good psychometric properties. The educational programme met high acceptability and proved to be effective in introducing changes in the physicians' perceived readiness as well as their perceived and actual knowledge.

Significance International experience/tools were tailored for Greek primary care services, demonstrating the great potential of comprehensive efforts to address IPV at the very early stage of contact of victimised patients with health care system. 